\title{
El gobierno urbano: indagaciones alrededor de las heterotopías innovadoras. Caso Medellín*
}

$\begin{aligned} & \text { Fecha de recepción: } 3 \text { de julio de } 2013 \\
& \text { Juan Esteban de aceptación: } 13 \text { de marzo de } 2014\end{aligned}$
\begin{tabular}{l|l|l|} 
Politólogo, Universidad Nacional de Colombia & Maestrando en Historia, investigador del grupo Narrativas Modernas \\
\hline y Crítica del Presente, Universidad Nacional de Colombia & jeposad0@unal.edu.co, jues80@gmail.com \\
\hline
\end{tabular}

Resumen El artículo se ocupa de las heterotopías y de cómo se convierten en una institución efectiva. Además, como el presente de las dinámicas económicas y sociales en Medellín se fundamentan, en gran medida, en un conjunto de acciones y políticas que responden al gobierno individual. En este contexto se analiza la señal que la razón gubernamental intenta emitir con éxito aparente, idea esta que promueve a Medellín como un buen "producto-ciudad" que, conocedora de sus limitaciones, intenta iniciar procesos para reducir las profundas desigualdades sociales que la destacan, pero fracasa en el intento. Por lo tanto, se intentará dar cuenta de que la heterotopía, obviamente responde a sus lógicas normativas y físicas, basadas en la necesidad de asegurar la solidaridad, incluso mediante la invitación a establecer un orden ilegal.

Palabras clave Ciudad; heterotopías; utopías

Palabras clave Espacio urbano; espacio en arquitectura; Medellín, Colombia descriptor

Una primera versión de este artículo se presentó en el Seminario de Investigación Espacio y Modernidad, desarrollado en Medellín entre febrero y junio de 2013. Forma parte de los resultados de indagación y reflexión que se vienen dando en el marco del desarrollo de la tesis de Maestría en Histo ria, Historia de dos ciudades imbricadas. Medellín ciudad utópica y heterotópica 1950-2010, para la Universidad Nacional de Colombia, realizado en e grupo Narrativas Modernas y Crítica del Presente, reconocido por Colciencias con clasificación B, adscrito a la misma Universidad. 


\section{Urban Governance: Inquiries About Innovative}

Heterotopias. Medellin Case

Abstract The article deals with heterotopias and how they become an effective institution. Furthermore, how the present economic and social dynamics in Medellin are based largely on a series of actions and policies that respond to individual government. In this context it is analyzed the signal that the government tries to convey with apparent success, this idea depicts Medellin as a good "city-product" which, aware of its limitations, tries to start processes to reduce the profound social inequalities that stand but fails in the attempt. Therefore, it will be analyzed that the heterotopia obviously responds to its logical and physical standards, based on the need to ensure solidarity, even by encouraging the establishment of an unlawful order.

Key words City; heterotopias; utopias

Key words Urban space; space (architecture); Medellín, Colombia plus

\section{0 governo urbano: indagações ao redor das heterotopias inovadoras.Caso Medellín}

Resumo $\mathrm{O}$ artigo ocupa-se das heterotopias e como elas mudam para uma instituição efetiva. Aliás, como o presente das dinâmicas econômicas e sociais em Medellín são fundamentadas, em grande medida, no conjunto de açóes e políticas que respondem ao governo individual. Neste contexto analisa-se o sinal que a ração governamental tenta emitir com bom sucesso aparente, ideia que visa promover Medellín como um bom "produto-cidade" que, conhecedora das suas limitaçóes, visa iniciar processos para reduzir as profundas desigualdades sociais que a fazem destacar, mais fracassa na tentativa. Por tanto, o objetivo é dar conta de que a heterotopia, obviamente, responde a suas lógicas normativas e físicas, baseadas na necessidade de assegurar a solidariedade, mesmo através de convite para estabelecer uma ordem ilegal.

Palavras chave Cidade; heterotopias; utopias.

Palavras chave Espaço público; espaço (arquitetura); Medellín, Colombia descritor 


\section{Introducción: impugnaciones míticas y reales}

Michel Foucault (2010) utiliza y presenta los "contraespacios" o heterotopías, relaciones que se han madurado y se han evidenciado como moldeadoras del contexto urbano en donde se encuentran. Las heterotopías, en cuanto a la intención real de su naturaleza impugnante, impactan en el espacio de la vida urbana y evalúan la capacidad de vivir en aislamiento, en crisis, en constante desviación, en permanente cambio, en insistente yuxtaposición de singularidades como los cierres y las aperturas, como las ilusiones y las realidades.

Así, vemos que la toma del contexto urbano, por parte de la heterotopía, evidencia que hay elementos que no permiten a la "utopía-ciudad" estructurar una lógica que materializa, una intención de ciudadanizar, una transición cognitiva, política y económica, una razón gubernamental efectiva, eficiente y eficaz.

Es oportuno tener presente que la fuerza de una heterotopía de crisis —aquella que se toma el contexto urbano- - surge de la definición lefebvriana que reviste de realidad las contradicciones constantes, los cambios o las yuxtaposiciones temporales y espaciales en la vida urbana, no menos institucionales de lo que se podría imaginar, pues dicha esfera — la vida urbana — es una composición espacial definida en Lefebvre por demografías, psicologías y geografías; una aglomeración urbana-humana densa y heterogénea que, aunque inestable, conforma instituciones que promueven directrices sociales y formas de vigilancia. Podríamos decir que las heterotopías son, en efecto, el sentido de reelaboración constante de las definiciones, de las propiedades, de los tiempos y los espacios de la vida urbana, impugnaciones que se manifiestan con el rigor de lo adverso (Harvey, 2013, p. 15) ${ }^{1}$.

En este contexto, la heterotopía se convierte en una institución efectiva, dado que es un escenario que realiza cambios graduales, pero profundos, en y desde el individuo, o sea, estructura una dinámica de carácter ético-político. Esta dinámica puede ser desfavorable a los códigos morales-legales, puesto que el mismo escenario urbano es desfavorable, porque la propia supervivencia depende de conquistar la esfera material que permite la inserción en el mercado (Bauman, 2007, p. 78$)^{2}$. Por otra parte, sigue siendo una incógnita

1 El concepto lefebvriano de heterotopía (radicalmente diferente del de Foucault) delinea espacios sociales fronterizos de posibilidad donde "algo diferente" no solo es posible sino básico para la definición de trayectorias revolucionarias. Ese "algo diferente" no surge necesariamente de un plan consciente, sino de lo que la gente hace, siente percibe y llega a articular en su búsqueda de significado para su vida cotidiana. Tales prácticas crean espacios heterotópicos en todas partes.

2 Para Bauman, la sociedad de consumidores es un tipo de sociedad que interpela a sus miembros en cuanto a su capacidad de consumidores. Al hacerlo de este modo, la sociedad espera ser escuchada, atendida y obedecida. Evalúa — recompensa y penaliza — a sus miembros según la rapidez y calidad de su respuesta a dicha interpelación. Como resultado, los lugares ganados o asignados sobre el eje de excelencia/ineptitud de rendimiento consumista se convierten en el principal factor de estratificación y en el criterio fundamental de inclusión y exclusión, a la vez que marca la distribución de la estima o el estigma social, así como la cuota de atención pública. 
hasta qué punto la disposición de la razón gubernamental, que pretende realizar la utopía de "los cambios profundos en la estructura social y económica de la ciudad", producirá una "ciudad" viable y dinámica, desde el punto de vista de esta inserción en la lógica del mercado. Por ende, la vida urbana (Posada, 2013, p. 20) ${ }^{3}$, se traduce en victorias o derrotas de y en la "ciudad" para cada individuo (Delgado, 1999, p. 85) .

Se dice que la crisis que se presenta -0 la heterotopía que resulta - parte de la desconfianza de los individuos en que podrán satisfacer sus anhelos (Bauman, 2007, p. 63) . El problema está en que el anhelo claramente se reduce, de forma sostenida, a su materialización; si no se concreta, se pierde la capacidad de inserción pública tan importante para alcanzar el estatus de ciudadano.

La heterotopía, entonces, dialoga para crecer, para incluir, para democratizar, para conquistar el desarrollo económico y la justicia social, conquista que puede ser legal o ilegal (Morey, 1986 , p. 23$)^{6}$. El desarrollo económico ilegal, por ejemplo, hace que las percepciones de riesgo caractericen negativamente, en un determinado espacio o tiempo, la acción heterotópica (Harvey, 2000, p. 183) ${ }^{7}$; así, no se puede establecer una confianza básica y una expectativa positiva en la vida urbana, aquella que está por fuera de la comprensión utópica llamada "ciudad".

Por ejemplo, desde el punto de vista de la inmediata relación utopía-heterotopía y además como archipiélago de la vulnerabilidad externa (Soja, 2008, p. 436) ${ }^{8}$, el centro de Medellín propone y mantiene un conjunto de acciones destinadas a concretar responsabilidades y confiabilidad como espacio institucionalizado, como máximo órgano de la heterotopía ilegal.

En síntesis, no son pocas ni irrelevantes las alternativas de acción frente a la profunda vulnerabilidad externa, como opciones para el quehacer urbano. Cabe preguntarnos por la coherencia entre el discurso utópico llamado "ciudad de Medellín" y las políticas urbanas, las estructuras sociales, las dinámicas heterotópicas de la vida urbana. Hay que ver, pues, la estrategia del Gobierno heterotópico, concebida como un instrumento para

3 El autor se plantea que en el espacio, en su ambigüedad, la contradicción y las evocaciones materiales se agrupan varias ideas, donde la realidad está sujeta a la noción de permanencia y de mantenimiento de puntos de significación, al considerar la libertad como poseedora de un sistema complejo de significaciones y ser la única alternativa de acción para diferenciarse de los demás, porque hace existir una enunciación para las desigualdades. En este contexto, las experiencias perturbadoras, laborales, incapacitantes, etc. y cuanto ente de socialización esté inmerso en la percepción formadora de lo público y lo privado, estructuran un concepto con múltiples apreciaciones; sin embargo, en torno a la libertad legal existe una cierta convergencia abierta de ideas y razones. Las estigmatizaciones y la libertad pueden resultar un aprendizaje y también pueden mejorar la comprensión de la importancia de la vida urbana.

4 Delgado, en el Animal público, muestra que son distintas las maneras mediante las que determinados grupos pueden reclamar su derecho a usar de manera expresiva el espacio público urbano y otorgarle un determinado significado compartido a las calles, las avenidas o las plazas por donde circulan o en las que se concentran. Las formas que este sentido del espacio público ha adoptado pueden ser diferenciadas a partir de una tipología siempre relativa, en la medida en que sus expresiones experimentan una constante tendencia a la superposición y a la mixtura: bandas, combos, tribus urbanas, etc.

5 Para Bauman es simple y llanamente el objeto de deseo consumista.

6 Morey, al invocar el análisis que hace Deleuze, habla de que Foucault desmonta una complicidad entre poder y Estado, como si se hubiera roto. Dice que Foucault no se contenta con decir que hay que repensar determinadas nociones; ni siquiera lo dice, lo hace y propone así nuevas coordenadas para la práctica. En el trasfondo ruge una batalla, con sus tácticas locales y sus estrategias de conjunto, que no proceden por totalización, sino mediante relevadores, empalmes, convergencias y prolongaciones.

7 David Harvey, en sus Espacios de esperanza, aborda brevemente el concepto de acción heterotópica. Sugiere que en la conferencia de Foucault "el problema de la utopía podía ser resucitado y simultáneamente evadido". A pesar de la referencia de Foucault a cárceles, hospitales, hogares de ancianos, museos y cementerios, Harvey también sugiere que hay que "escapar" de la cuenta de Foucault. Se pide la noción de "ordenar una alternativa" al sugerir que estos espacios permiten que "el otro" florezca. Reconoce algunos de los puntos fuertes de la noción de Foucault al destacar las diversas formas utópicas materiales que han surgido y que no son "mutuamente excluyentes" y celebra los espacios donde "la vida se experimenta de manera diferente" y, en general, insiste en la "heterogeneidad del espacio". Sin embargo, en la construcción de una crítica de la heterotopía, Harvey ataca a Foucault por ser un tanto ingenuo: "En muchos de mis ensayos sostengo que la noción de heterotopía de Foucault desafía y perturba, pero no presenta una idea de una alternativa, el utopismo espacio-temporal".

8 En su Postmetrópolis, Soja define archipiélagos como espacios protegidos y fortificados, islas de confinamiento y de protección preventiva contra los peligros tanto reales como imaginarios de la vida diaria; además, plantea que es en el centro de la ciudad donde la encarnación física de un espacio vigilado y carcelario es más evidente para ejemplificar el archipiélago, contemplado desde afuera como el foco generador y más popular que puede vulnerar los paraísos de seguridad y el estilo de vida de los demás archipiélagos de la ciudad. 
viabilizar transformaciones económicas y sociales en los archipiélagos de Medellín.

\section{Archipiélago e identidad}

Como fundamento de un conjunto de acciones y políticas que responden al gobierno individual, el planteamiento de las dinámicas económicas y sociales en Medellín pretende ejemplificar el hecho de que la planeación urbana, las políticas públicas legales y la educación ciudadana son cada vez menos importantes para interpretar los complejos procesos que caracterizan las relaciones urbanas contemporáneas, escenarios donde los individuos toman decisiones basados en la efectiva materialización de deseos consumistas (Bauman, 2007, p. 118)9 . El escaso margen de maniobra que caracteriza la utopía-ciudad para promover cambios en la estructura de la economía y, en consecuencia, en la sociedad, la aísla como objeto de promoción en el mercado, como órgano de alianzas sociales, como primer laboratorio de vida social y como institución en el campo abierto de las relaciones sociales extensas.

Esta consecuencia se reafirma con la intención manifiesta de profundizar la desintegración de la legalidad y se concreta en la conjugación del conjunto completo de estructuras ilegales que brindan seguridad, dinamizan la economía, promueven el empleo, dan vivienda, etc. ${ }^{10}$, estructuras forjadas fuera de la utopía-ciudad que permiten la accesibilidad a derechos fundamentales en la atmósfera de la vida ilegal, espacialmente, como archipiélagos.

Tales archipiélagos fragmentan la vida urbana desde la creación de unos conectores entre instituciones ilegales, o sea, una configuración y codificación de la norma. Por ejemplo, la muerte como máxima innovación heterotópica define el límite, el castigo y la contravención en el archipiélago, como espacio de formación del individuo político y normalizado y campo urbano de aplicación viable de lo aprendido (Personería de Medellín, 2013, p. 32) ${ }^{11}$.

Estos conectores posibilitan la materialización de los deseos y la normalización de las instituciones e individuos en y para un espacio. Dicha normalización puede ser interpretada como una señal genuina del deseo satisfecho, énfasis de consolidación que tiene el claro objetivo de profundizar la interdependencia, por medio de la integración física y normativa de los individuos.

En este contexto, se puede deducir que el acontecer de la utopía-ciudad se basa únicamente en la necesidad de asegurar el respeto al orden público, sin priorizar órdenes previos como el económico, el social o el alimentario, por ejemplo. En contraposición, la heterotopía urbana implementa en el archipiélago la priorización de los órdenes olvidados por la utopía-ciudad, sin importar si son conseguidos por medio de la ilegalidad.

9 Para Bauman, el tema no es elegir en sí, pues uno debe hacerlo bajo pena de exclusión, aunque no tenga la libertad de modificar de algún modo las opciones disponibles; no hay otras alternativas, ya que todas las posibilidades realistas y aconsejables han sido preseleccionadas, preescritas y prescritas.

10 En lo que respecta a los actores señalados, en su mayoría son grupos armados ilegales de incidencia directa en los entornos de alta conflictividad de la ciudad; se constituyen espacios donde se configura una forma de amenaza general, impersonal y abstracta, pero igual o más efectiva que la amenaza directa. Estos entornos amenazantes son una forma de gobernabilidad que materializan la vida urbana y de heterogeneidad natural e imbrica Gobierno y ejercicio del poder, con la producción de espacio.

11 Según la Meval, en el Área Metropolitana 302 estructuras delincuenciales emplean la microextorsión como renta criminal. Con falsos compromisos de seguridad, amenazas de muerte 0 atentados contra la vida, presionan a sus víctimas a ceder ante sus exigencias. En 2012, por ejemplo, ocho conductores de buses fueron asesinados por no pagar vacuna. En noviembre, el gerente de Camacol denunció que varios proyectos de viviendas estaban suspendidos por problemas de extorsión. La Policía registró 126 casos de extorsión en el año, de los cuales 32 fueron con medios de transporte, 36 con comerciantes, 4 con empresas de construcción y 54 con ciudadanía en general. Por su parte, la Dirección Seccional de Fiscalías de Medellín recibió 607 denuncias por este delito. Los barrios con mayor afectación extorsiva según la Meval son Doce de octubre, Picacho, Robledo, Aures, San Javier, La Loma, La Pradera, Santo Domingo, Granizal, Santa Cruz, Santa Rita, San José de la Cima, La Sierra, Caicedo y el centro de la ciudad. 
Así, si bien no existe la intención consciente de participar de manera activa en la actividad ilegal de la heterotopía, el individuo puede enviar una clara señal de que está dispuesto a asumir responsabilidades en ese escenario urbano y consolidar su identidad y su comportamiento en el orden público heterotópico, lógicamente orientado por los intereses vinculantes de "la sociedad de consumidores" (Bauman, 2007, p. 77).

Pero la concreta inclinación ideológica de la razón gubernamental (Foucault, 2007, p. 28) ${ }^{12}$, en el plano del discurso, hace que se denuncie la dicotomía utopía-heterotopía y se reafirmen los flujos de instituciones vinculadas a la acción ilegal o legal, como entes que contextualizan. En otras palabras, el grupo que formula la política pública legal (Alcaldía de Medellín) piensa en términos de una estructura dividida entre buena y mala ciudadanía, proceso excluyente que favorece y consolida regímenes ilegales que organizan y estructuran en la ciudad redes de bandas, combos, pandillas, etc. ${ }^{13}$. Ello ocurre, en parte, porque se reconoce de manera implícita que las estructuras de integración urbanas son convenientes para el equilibrio de la inserción social.

\section{El equilibrio, entre la exclusión y la fijación}

Así pues, encontramos el sentido del conector, aquel que ha permitido el ideal propuesto por los prototipos estatales. El desarrollo del conector se fundamenta en la señal que la razón gubernamental intenta emitir con éxito aparente y que consiste en la idea de que Medellín es un buen "producto-ciudad", que es conocedora de sus limitaciones e intenta iniciar procesos para reducir las profundas desigualdades sociales que la destacan. Así, el gobierno institucional legal de la "ciudad" confiere a la política de seguridad un papel cada vez más central en la promoción del desarrollo económico y social (Plan de Desarrollo 2012-2015, p. 18) ${ }^{14}$.

Es oportuno tener presente que la razón gubernamental legal en los últimos años se encaminó, en realidad, hacia el centro del debate sobre la seguridad, incluso por tener la conciencia de que solo tendría condiciones de mantener la institucionalidad si construía una agenda social vinculante. Esta decisión firmó un compromiso de promocionar cambios graduales en la redistribución de la seguridad.

Dicha paradoja entre vincular y dar seguridad contiene la propia supervivencia de la razón gubernamental, sobre todo la ilegal, ya que hace emerger la capacidad para gobernar y para conquistar la confianza de la comunidad en su labor de producir un "individuo viable y dinámico" desde el punto de vista de su inserción en la economía.

En este contexto, la razón gubernamental legal pretendió convencer al ciudadano medellinense de que la vida, la equidad, la educación, el desarrollo sostenible y los buenos gobiernos,

\footnotetext{
12 Foucault plantea que es una limitación de hecho que se plantea en función de la práctica gubernamental; es una división entre lo que es preciso hacer y lo que conviene no hacer. Esta división no se establecerá en los individuos sino en el dominio mismo de la práctica gubernamental o, mejor, entre las cosas por hacer y los medios que deben emplearse para hacerlas, por un lado y las cosas que no hay que hacer, por otro.

13 Desde las organizaciones civiles, como la Corporación para la Paz y el Desarrollo Social en Medellín (Corpades), las cifras hablan de unos 350 combos con entre 8.000 y 10.000 miembros, conformados en un 40\% por menores de edad.

14 El Plan de Desarrollo 2012-2015 "Medellín, un hogar para la vida" se fundamenta en principios claros y contundentes que le dan fuerza, alma y contenido a cada programa y proyecto público: la vida es el valor supremo, todos nuestros esfuerzos deben dirigirse a protegerla, honrarla y valorarla y nada puede vulnerarla sin que nos movilicemos a defenderla. Transparencia y buenos Gobiernos que actúen y construyan bajo una cultura de legalidad y la activa construcción de una sociedad participante son la base fundamental para el desarrollo democrático. Estas son las claves para la construcción de la Medellín del siglo XXI.
} 
fundamentos del Plan de Desarrollo 2012-2015, se vinculan en decidido apoyo con las organizaciones sociales, empresariales y académicas y apuntan a un modelo de "ciudad" que prioriza la seguridad como herramienta de transformación social, de creación de oportunidades, de superación de la inequidad y el desarrollo de capacidades y habilidades para la sociedad del siglo XXI, de ciudadanos globales, solidarios, pacíficos, respetuosos de los otros y del ambiente.

Contrario a la utopía anterior, al tiempo que la vida urbana contribuye al aumento de la percepción de riesgo asociado con los archipiélagos, alimenta la desesperanza, dada la incapacidad de materializar los postulados del Plan de Desarrollo; con ello obliga a la razón gubernamental legal a elevar las estrategias internas, por ejemplo, entregar el orden público a la institucionalidad ilegal. Esto podemos hallarlo en los datos que nos entrega la Personería de Medellín, cuando expresa que, en 2012, el riesgo de los seres urbanos estuvo visualizado en el centro de la ciudad con la tasa de homicidios más alta: "Las comunas que tuvieron una mayor tasa de homicidios fueron en su orden la comuna 10 (Candelaria), 13 (San Javier), 8 (Villa Hermosa), 4 (Aranjuez), 15 (Guayabal) y 16 (Belén). 11 casos de asesinato a las afueras de las instituciones escolares" (Personería de Medellín, 2013, p. 18).

Mientras la mayoría de los programas de la razón gubernamental legal reiteraban recursos coercitivos y se aislaban de la vida urbana, esta misma llegó a generar estrategias de pacificación desde las expresiones delincuenciales más extremas, como la muerte ${ }^{15}$.

La búsqueda del equilibrio por parte de la razón gubernamental legal se materializa en cifras positivas para convencer a la sociedad de que no solo el Plan de Desarrollo se ha madurado, sino también para evidenciar su capacidad de Gobierno, lo cual tiene sentido. Lo que no se dice es que la crisis que se presenta resulta, en gran medida, de la desconfianza de los individuos en relación con la intención real de la razón gubernamental legal de implementar los compromisos asumidos de manera pública.

Si llevamos el raciocinio hasta sus últimas consecuencias, se concluirá que a partir de la utopía del Estado (políticas, planes, programas, etc.) y por medio de sus instituciones, o sea, lo político, se establece una normalidad y una naturalización del buen y mal ciudadano, contrario a los cambios políticos y de la realidad política de la vida urbana, que se presentan de forma violenta en la efervescencia heterotópica como victoria de la razón gubernamental ilegal, seleccionada entre diversas opciones políticas ${ }^{16}$.

\section{¿Heteronomía, praxis y consecuencia?}

La discusión que fundamenta este escrito tiene que ver con el inicio de los cambios urbanos que se generan en unas acciones fundamentadas en

15 El pie de página dice: De acuerdo con los datos ofrecidos por la fuente, en la ciudad fueron asesinadas en promedio 104 personas por mes, 24 en la semana y 3 cada día y la tasa de la ciudad fue de 52 homicidios por cada cien mil habitantes (hpcmh), cifra que constituye una de las tasas más altas de las ciudades capitales del país, en comparación con Bogotá con una tasa de 16 hpcmh (El Tiempo, 2013), Cali con una tasa 82,2 hpcmh (El País, 2013) y Barranquilla con una tasa de 29,1 hpcmh (El Tiempo, 2013), mientras la del país es de 31 hpcmh (El Nuevo Siglo, 2013). En general, en el país se presentó una tendencia a la baja de los homicidios, pero Medellín aún ostenta una tasa alta.

16 Por ejemplo, el incremento de la presencia criminal en la periferia y algunas zonas urbanas de Medellín, así como de fenómenos violentos como la desaparición y el desplazamiento forzados se mantienen como focos de denuncias de organizaciones sociales y de derechos humanos. Así lo demuestra el informe de las ONG Corporación para la Paz y el Desarrollo Social (Corpades), Instituto Popular de Capacitación (IPC), Corporación Itagüí Nueva Gente, Movimiento Ríos Vivos y la Asociación Campesina del Norte de Antioquia. Los grupos ilegales logran establecerse en la periferia de la ciudad, "en zonas bases que son San Antonio de Prado, la parte alta de Altavista y San Cristóbal, Pan de Azúcar y el corregimiento San Félix en San Pedro de los Milagros”. Desde esos sectores disputan el control de zonas como el occidente de Medellín que representan rentas por extorsión, microtráfico, tráfico de armas y hurto de combustibles. "Producto de esa disputa son las continuas balaceras en zonas como la comuna 16 de Belén, la 13 y la 8. La desaparición forzada de personas, lo que se evidencia en fosas en la ciudad y hallazgo de cadáveres en el río Medellín. El desplazamiento forzado intraurbano no cede y prueba de ello fue la salida masiva de cerca de 100 familias de San Cristóbal en mayo pasado" (Personería de Medellín, 2013, p. 17) 
un ejercicio grupal, heteronómico, o sea, en una efectiva aplicación de las normas que regulan la acción del sujeto; el individuo no queda regulado por una conciencia, sino por algo ajeno a ella, una total oposición a la autonomía. Dicha heteronomía política se materializa en el hecho de que la distinción entre "ciudad" y vida urbana es cada vez más importante para interpretar las relaciones intrasocietales que caracterizan nuestro tiempo.

Esta discusión no es nueva, pero se ha tornado mucho más sistemática y sofisticada. Parte de cómo la implementación de un conjunto de heterotopías puede reducir la vulnerabilidad externa en la vida urbana y dilucida también cómo el mercado y la administración generan cambios en la estructura interna del archipiélago. Estos cambios se reafirman con acciones simbólicas y con acciones concretas como la decisión de implantar identidades, normas, instituciones, dinámicas económicas, ordenaciones de lo público, etc. La intención manifiesta de profundizar la integración heterotópica se concreta en la coordinación de prácticas que culminarían, si el discurso se transforma en acción, en individuos que tienen una identidad estructurada en el archipiélago y una identificación con su vida urbana.

La integración entre estos individuos se puede interpretar como una señal genuina del interés de "pertenecer" y como manifestación de un accionar político, pues se les "extiende" la mano en un momento en que los abandonos, la invisibilización, la exclusión, la fijación como amenaza y la vulneración de sus derechos, por parte de la utopía, los avasalla (Personería de Medellín, 2013, p. 61) $)^{17}$.
De otro lado, el énfasis en una estrategia de consolidación de la identidad tiene el claro objetivo de profundizar la integración y la interdependencia; si bien dicha necesidad de agruparse no es novedosa, las innovaciones se expresan en su disposición de asumir los costos por medio del enfrentamiento a disposiciones de tipo institucional legal.

Por lo tanto, la prioridad conferida a la utopía por parte del discurso oficial se evidencia en una disminución del interés en la misma, la cual depende de un aumento del interés en la heterotopía, como obvia respuesta a sus lógicas normativas y físicas, basadas en la necesidad de asegurar la solidaridad, incluso mediante la invitación a establecer un orden.

En este caso, la heteronomía comanda la acción; la indiscreta y profana autodestrucción de las inclinaciones ideológicas de la institución ilegal en el plano individual y social denuncia y reafirma las críticas a los flujos de capital humano (Foucault, 2007, p. 134), a las contradicciones del "deber ser" de la "ciudad" y a las injusticias del mercado.

Es así como la interdependencia entre las identificaciones y la simultánea consolidación de las identidades organiza geográficamente las heterotopías y estructura redes de normas propias y praxis particulares.

Por lo tanto, si el discurso y las actitudes a favor del fortalecimiento de las instituciones primarias no llaman mucho la atención, porque son formas tradicionales no prácticas para la inserción apta en el mercado, no se puede negar el serio riesgo de que esta participación en grupos que reemplazan a las instituciones primarias, en

\footnotetext{
17 Para poner un ejemplo que dé cuenta de la idea que se pretende esbozar, podemos hablar de unos datos obtenidos en entrevistas a algunos comerciantes informales. Se pudo establecer que su actividad laboral también se encuentra mediada por el pago de "extorsión" o pago a las "Convivir" y que los montos de este pago varían según el sitio y la mercancía que se venda, información que se encuentra precisada en un estudio realizado por la Universidad Nacional, en donde se refiere que "aunque la investigación no se haya centrado en aspectos como las mafias, sí se identificó que hay grupos que controlan el espacio público en Medellín y cobran un impuesto a ciertos venteros, con mecanismos de préstamos o con los conocidos "gota a gota", que funcionan con el préstamo de cierta cantidad en el día para desarrollar la actividad comercial y, al final de la tarde, regresan a cobrar hasta un $20 \%$ más del valor inicial.
} 
combinación con las praxis ilegales, signifiquen una modificación material de la vida urbana, una praxis social, como regla del juego para asegurar un "triunfo económico".

\section{Conclusión: demanda particular de ensueño}

Sumida en el orden temporal, ninguna estructura está a salvo de la corrosión del tiempo o de los acontecimientos imprevisibles; entonces, debe ser leída en su capacidad de hacer frente a lo inesperado. La heterotopía brinda la innovación a partir de los datos irreductibles de lo vivencial. Entonces, la heterotopía siempre es posible si cambia con el tiempo, al mismo ritmo, veloz y estrepitosa.

Sobre el modelo que ofrece la vida urbana, como tipo de la inestabilidad vivencial, como un régimen más conforme a la naturaleza de lo humano, como división, como corrosión, como corrupción que se entrevera, que se divide en dos universos a la vez irreconciliables y simétricos, como sentido de la vida, como fundamentado en la dinámica urbana, como la mirada particular, ligada a la "verdad" y a la "mentira", con todas sus marcas, responsabilidades y necesidades, se encuentra la ética de los hechos, el peso de lo real.

En este contexto, el gobierno urbano planea y participa desde el imperativo heterotópico, escenario en el cual las acciones del Ejecutivo se pierden en actos deshilvanados, sin armonía o complementación. La sociedad, sin la vida urbana y sin la institucionalización de la heterotopía, no tendría un punto de referencia a dónde acudir para darle orden y sentido a sus propias iniciativas.

Por lo tanto, la "ciudad" no es más que un estéril ejercicio retórico, una acción estatista que sustituye a la sociedad e inhibe su creatividad. No permite la atención eficaz de las prioridades que democráticamente se han propuesto en lo real de la vida urbana. Cuando hablamos de lo real, nos referimos a un esfuerzo de previsión, racionalidad, orden, coordinación y, sobre todo, un gran trabajo de conciliación entre el interés de los individuos y los de la sociedad, entre el pasado y el futuro.

Se puede decir que las heterotopías son lugares, redes, entornos, servicios urbanos y rutas para la inclusión como factor de gobernabilidad y seguridad. En segunda instancia, conciben y consideran el impacto en la totalidad del territorio, pero con especial empeño en aquellos territorios que sobresalen por tener bajos índices de seguridad, desarrollo humano y calidad de vida utópica.

En este contexto, las heterotopías se justifican en la medida en que son un propósito deliberado e innovador que implementan nuevas condiciones y dinámicas de inclusión social. Son una manera de conocer y apoyar la recuperación del territorio y la institucionalidad pública (Posada, 2012, p. 159), al promover la creación del disfrute de los bienes urbanos y cualificar el escenario en la diversidad de los asuntos para la imbricación utopía-heterotopía llamada Medellín.

\section{Bibliografía}

Bauman, Z. (2007). Vida de consumo. Fondo de cultura económica.

Delgado, M. (1999). El animal público. Barcelona: Anagrama.

Delgado, M. (2011). El espacio público como ideologia. Madrid: Catarata.

Foucault, M. (2007). Nacimiento de la biopolítica. Buenos Aires: Fondo de Cultura Económica. 
Foucault, M. (2010). El cuerpo utópico. Las heterotopias. Buenos Aires: Nueva visión.

Harvey, D. (2000). Espacios de esperanza. Madrid: Ediciones Akal.

Harvey, D. (2013). Ciudades rebeldes. Madrid: Akal.

Lefebvre, H. (1976). Espacio y politica. El derecho a la ciudad II. Barcelona: Ediciones Península.

Morey, M. (1986). Lectura de Foucault. Madrid: Taurus.
Personería de Medellín. (2013). Informe anual sobre situación de derechos humanos en Medellin. Medellín: Autor.

Posada-Morales, J. (2012). El espacio, la verificación de la soledad. Bitácora Urbano-territorial, 21 (2), 157-163.

Posada-Morales, J. (2013). La pobreza: consumo de identidad social en la ciudad. Revista de Urbanismo, 15 (28), 17-26.

Soja, E. (2008). Posmetrópolis. Madrid: Traficantes de sueños. 